\title{
Las cadenas globales de producción industrial en América Latina desde una perspectiva estructuralista
}

\author{
Carolina Teresita Lauxmann* \\ Manuel Facundo Trevignani** \\ Víctor Ramiro Fernández ${ }^{* * *}$
}

Fecha de recepción: 5 de agosto de 2020

Fecha de aprobación: 13 de octubre de 2020

Resumen: A partir de la recuperación de los principales conceptos del estructuralismo latinoamericano, este trabajo busca poner en discusión las potencialidades y limitaciones del nuevo paradigma de desarrollo de la actualidad, el enfoque de cadenas globales de valor, que desde comienzos de este siglo ha tenido una importante influencia tanto en la comunidad académica como en los organismos internacionales. Para ello, se analiza la agudización de las características periféricas de las estructuras productivas latinoamericanas de las últimas décadas, asociadas a su inserción en las redes de producción global y se contrastan con las experiencias exitosas de transformación estructural que en este mismo escenario han podido consolidar algunos países de Asia del Este. De dicho análisis se desprenden elementos para repensar la importancia de la industrialización para el desarrollo y las políticas necesarias para impulsarla en el actual escenario de cadenas globales de producción.

Palabras clave: industrialización; crecimiento económico; desarrollo económico; cadenas globales de valor; América Latina.

Clasificación JEL: F02, L16, L52, O10, O20.

Cómo citar este artículo:

Lauxmann, C., Trevignani, M., \& Fernández, V. (2021). Las cadenas globales de producción industrial en América Latina desde una perspectiva estructuralista. Apuntes del Cenes, 40(71). Págs. 75 - 101. https://doi.org/10.19053/01203053.v40.n71.2021.11556

* Doctora en Ciencias Sociales. Docente-investigadora de las facultades de Ciencias Económicas y de Humanidades y Ciencias de la Universidad Nacional del Litoral, Santa Fe, Argentina. claux@unl.edu.ar iD https://orcid.org/00000002-3959-0701. Contacto de correspondencia

** Candidato a doctor en Estudios Internacionales. Magíster en Relaciones y Negociaciones Internacionales. Docente-investigador de la Facultad Ciencias Económicas de la Universidad Nacional del Litoral, Santa Fe, Argentina. mtrevignani@hotmail.com (iD https://orcid.org/0000-0002-7428-2739

*** Doctor en Ciencia Política. Investigador independiente del Consejo Nacional de Investigaciones Científicas (CONICET). Docente de la Facultad de Humanidades y Ciencias de la Universidad Nacional del Litoral, Santa Fe, Argentina. rfernand@fcjs.unl.edu.ar (DD https://orcid.org/0000-0002-8650-8934 


\title{
Global Industrial Production Chains in Latin America From A Structuralist Perspective
}

\begin{abstract}
Recovering the main concepts of Latin American structuralism, this article examines the potentialities and limitations regarding the new development paradigm, the Global Value Chain approach, which over the last two decades has had an important influence among academic community and within international organizations. To achieve this, the authors analyze the exacerbation of the peripheral condition of the Latin American productive structures associated with their insertion in global production, contrasting them with the successful experiences of structural transformation of the East Asian countries. From the analysis, the authors draw some elements to rethink the importance of industrialization for development, and the policies needed to promote it in the current scenario of global production chains.
\end{abstract}

Keywords: industrialization; economic growth; economic development; global value chains; Latin America. 


\section{INTRODUCCIÓN}

Si bien industrialización no es sinónimo de desarrollo (Arrighi et al., 2003; Hirschman, 1968), la gran mayoría de los países que hoy conforman el centro de la economía mundial han atravesado por procesos de desenvolvimiento industrial que permitieron configurar una base manufacturera compleja y dinámica (Fernández, 2017; Fernández \& Lauxmann, 2014). Para los países de América Latina, sin desconocer los avances en términos de industrialización que han experimentado a lo largo de su historia (Bértola \& Ocampo, 2013), la constitución de un sector manufacturero con estas características continúa siendo una tarea pendiente.

En el marco de la reconfiguración capitalista iniciada en los años 70 , se presenta desde fines del siglo XX e inicios del XXI una alternativa relativamente "fácil" de industrialización a los diferentes países periféricos a través de su incorporación a las redes de producción global (Baldwin, 2011). Esta estrategia, según prestigiosos organismos internacionales (OI), se muestra promisoria para propiciar su desarrollo
(Blyde \& Trachtenberg, 2020; Banco Mundial, 2020). Incluso, algunos estudios sobre la reciente experiencia exitosa de un pequeño cúmulo de países localizados en Asia del Este para salir de la periferia global (Gereffi, 2005; Hamilton \& Gereffi, 2009), parecen reforzar las ventajas de la industrialización asociada a la participación en redes globales de producción.

Sin embargo, esta industrialización "por invitación", lejos de viabilizar el desarrollo de los países latinoamericanos, parece reeditar bajo nuevas formas viejos problemas identificados hacia mediados del siglo XX por algunos intelectuales aglutinados en la Comisión Económica para América Latina (CEPAL), que teorizaban sobre la conformación del sistema capitalista mundial jerárquico y desigual, configurado sobre la base de centros y periferias. En este sentido, consideramos que las recomendaciones de políticas para el desarrollo emanadas de los OI, particularmente aquellas que se sustentan en el enfoque que ha logrado mayor influencia dentro de los mismos: el de cadenas globales de valor, si bien pueden dar lugar a impulsar el desenvol- 
vimiento del sector manufacturero de algunos países de América Latina, lejos se encuentran de habilitar una salida de su posicionamiento periférico.

En consecuencia, el objetivo de este trabajo es poner en discusión la potencialidad de este nuevo enfoque de cadenas globales de valor, así como sus recomendaciones de política para propiciar el desarrollo de los países periféricos en general, y de la región latinoamericana en particular, apelando a los postulados centrales del estructuralismo latinoamericano. Para avanzar en este propósito, el artículo se divide en tres partes. En la primera se realiza una breve presentación de los diferentes marcos teóricos-analíticos que desde mediados del siglo XX han sido relevantes en materia de desarrollo, reparando particularmente en los planteamientos de la CEPAL, que prevalecieron entre las décadas de 1950-1970 en la región, y en el enfoque de cadenas globales de valor, que ha ido ganando peso desde inicios del siglo XXI. En dicho apartado se brindan elementos para analizar en la segunda parte la particular configuración de las estructuras productivas de América Latina entre 1990-2018 y evidenciar la reconfiguración de las condiciones de periferialización antes mencionadas, en el marco de la inserción en las redes de producción global. Sobre la base de dicho análisis, y contrastándolo con las experiencias exitosas de los países de Asia del Este, que a través de una inserción dirigida por el Estado en las cadenas de producción global han podido viabilizar procesos de desarrollo, avanzamos a la tercera parte. En ella, a modo de reflexiones finales, se abordan los límites de la estrategia de desarrollo basada en una industrialización por inserción en las redes globales de producción y la necesidad de recuperar, a la luz de este nuevo escenario productivo, los planteamientos centrales del estructuralismo latinoamericano para repensar estrategias de salida de la periferia global.

\section{LA PROBLEMÁTICA DEL DESA- RROLLO. CONTRIBUCIONES TEÓRICAS Y RECOMENDACIO- NES DE POLÍTICA ENTRE ME- DIADOS DEL SIGLO XX Y PRIN- CIPIOS DEL XXI}

La problemática del desarrollo comienza a circular ampliamente en los dominios de la ciencias sociales en el mundo hacia mediados el siglo XX, en el particular contexto de guerra fría entre Estados Unidos y la Unión de Repúblicas Socialistas Soviéticas, y de inicio del proceso de descolonización de África y Asia (Wallerstein, 1993). Llegó a constituirse como una subdisciplina importante de la economía, la cual abordaba las dificultades que se presentaban en determinados países -fundamentalmente aquellos que tras la Segunda Guerra Mundial fueron logrando la independencia- para alcanzar un crecimiento dinámico y sostenido del producto o renta nacional. Entre sus representantes más reconocidos podemos mencionar a 
Nurkse, Rosenstein-Rodan, Rostow, Lewis, Myrdal, los cuales llegaron a ser considerados como "los pioneros de desarrollo" (Bustelo, 1999; Svampa, 2016; Unceta Satrústegui, 2009). Estos teóricos consideraban que la diferencia entre los países subdesarrollados y los desarrollados no era tanto de grado, sino con respecto a estructuras. Dentro de este marco interpretativo, e influenciados por la teoría macroeconómica keynesiana, entendían que si el Estado impulsaba procesos de transformación de las estructuras productivas de los países subdesarrollados a través del fomento de la acumulación de capital, y más específicamente de la industrialización, se incrementaría dinámicamente la renta y el producto per cápita, y se desdibujaría la brecha existente con los países desarrollados (Bustelo, 1999).

El continente latinoamericano ocupó un lugar central dentro de la teoría económica del desarrollo. Con los precursores aportes de Prebisch, seguidos de las contribuciones de un cúmulo de intelectuales aglutinados en la CEPAL, durante los años cincuenta se fue conformando la escuela de pensamiento estructuralista latinoamericano. Esta escuela, si bien también apostaba a la transformación estructural a través de la industrialización impulsada por el Estado (CEPAL, 1951; Prebisch, 1949) y guardaba vínculos con la perspectiva keynesiana (Julio, 2008), presentaba diferencias con el cuerpo convencional de la doctrina. Para "los pioneros", la problemática del desarrollo era una cuestión meramente transitoria. Sus postulados estaban impregnados de una mirada evolutiva según la cual, si los países "subdesarrollados" seguían ciertas directivas, podrían alcanzar el desarrollo, en el marco del beneficio mutuo que ofrecía el esquema de relaciones económicas internacionales vigentes (Svampa, 2016). Por el contrario, para los teóricos de la CEPAL dicha transformación, lejos de estar garantizada, se presentaba obturada por la particular forma de articulación a la economía internacional (Nahón et al., 2006). Para comprender esta postura es necesario que nos explayemos en algunos de los planteamientos originarios del estructuralismo latinoamericano.

Uno de los primeros y principales aportes realizados por Prebisch, que pueden encontrarse en sus textos de finales de 1949, y verse reiterados muy poco después en un documento publicado a inicios de los cincuenta, se enfoca en la consideración de la relación centro-periferia. Esta se derivaba de entender la existencia de un sistema capitalista mundial, consolidado bajo la hegemonía británica, que presentaba, por un lado, un conjunto de países -centrales- en los que tempranamente se había desarrollado y difundido el progreso técnico al conjunto de la estructura productiva. Y, por otro lado, un grupo de países - periféricos- que se encontraban rezagados en términos de progreso técnico, en donde los escasos avances existentes en esta materia se encontraban fuertemente concentrados en ciertos 
sectores de su estructura productiva, fundamentalmente aquellos que se vinculaban con la extrovertida economía de Gran Bretaña (Guillén Romo, 2008; Rodríguez, 2006). El primer grupo de países estaba constituido por los grandes centros industriales, entre los que se encontraban en ese entonces Gran Bretaña, algunos países de Europa occidental, Estados Unidos y Japón. El segundo grupo de países, por su parte, estaba conformado por una amplia y variada periferia, que se vinculaba con aquellos atendiendo a sus necesidades, proveyéndole principalmente alimentos y materias primas a bajo costo.

Vemos así que la satisfacción de las necesidades de los centros - particularmente la del centro hegemónico- opera como principio ordenador del sistema capitalista mundial. Es decir, era a partir de la necesidad inicial de Gran Bretaña que los países periféricos podían establecer una vinculación, y es entonces esa necesidad del "hegemón" la que condicionaba, en gran medida, la inserción de la periferia en el sistema mundial y la estructura productiva interna de los diferentes países sobre la que se sustenta aquella. Las estructuras productivas internas de los países de la periferia, configuradas en el marco de esta particular relación con el centro, desarrollaron las características de especialización y heterogeneidad. Es decir, se conformaron economías que concentran el escaso progreso técnico existente en sectores dedicados a producir y exportar productos primarios que tienen por mercado de destino los países centrales. Y, a su vez, estos sectores presentan un marcado diferencial de productividad en relación con el resto de los sectores productivos de la economía (Rodríguez, 2006; Gurrieri, 2011).

Ante este escenario, podemos advertir entonces que la existencia de centros y periferias no responde solo a una situación de diferencias de origen en términos de generación y penetración del progreso técnico, sino que, dada la misma, se vincula al dinamismo de la expansión capitalista. La configuración de un sistema capitalista mundial jerárquico y desigual se encuentra asociada a la forma que asumen las estructuras productivas de los espacios periféricos en su inserción al sistema internacional y la capacidad de generación y difusión del progreso técnico que ello implica.

Esta particular forma de articulación de la economía mundial, analizada por Prebisch, que tendía a concentrar el progreso técnico en los centros industriosos del sistema capitalista y a relegar a una posición subordinada $\mathrm{y}$ dependiente a la periferia, requería ser atacada en sus fuentes si se pretendía alcanzar el desarrollo. Es decir, era necesario avanzar, según la CEPAL, en el estímulo de aquellas actividades representativas por excelencia del progreso técnico, en ese entonces, las industriales. De esta manera, a través de un proceso de industrialización, las economías periféricas podrían lle- 
var sus estructuras productivas hacia mayores niveles de diversificación $\mathrm{y}$ homogeneidad, disminuyendo la brecha tecnológica y de ingreso que existía con los países centrales.

Los planteamientos de la CEPAL tuvieron una profunda influencia entre los responsables del diseño y ejecución de las políticas económicas de la región (FitzGerald, 1998). De este modo, con posterioridad a la Segunda Guerra Mundial, tuvo lugar en diversos países latinoamericanos un proceso de industrialización dirigido por el Estado (Bértola \& Ocampo, 2013). Es necesario destacar que este tipo de estrategia de desarrollo orientada "hacia adentro", rompiendo con la dinámica vigente hasta el momento de desenvolvimiento traccionado por las exportaciones orientado "hacia afuera"-, se encontró favorecida por el cambio del centro hegemónico del sistema mundial. Bajo la hegemonía británica primó una estrategia de acumulación extrovertida, en la cual las pequeñas empresas especializadas y flexibles que dinamizaban las ramas más importantes de su actividad económica establecieron fuertes vínculos de complementariedad con las economías de los países coloniales y extranjeros para llevar adelante, articuladamente -bajo un régimen de fisión vertical-, los diferentes procesos productivos, dando un fuerte impulso al mercado mundial. Con la consolidación de Estados Unidos como centro hegemónico, esta estrategia de acumulación fue desplazada por otra de tipo auto- centrado, que implicó un proceso de "internalización del mercado mundial" en grandes empresas verticalmente integradas y burocráticamente administradas, que producían en masa para mercados domésticos relativamente cerrados (Arrighi, 1999).

Este cambio en la estrategia de acumulación del nuevo centro hegemónico dio lugar a modificaciones en el comercio internacional. El patrón de intercambio característico de la hegemonía británica, por el cual los centros producían y vendían bienes manufacturados a los periféricos, los que se abocaban a la producción y comercialización de bienes primarios, perdió vigencia. Con posterioridad a la Segunda Guerra Mundial, crecieron fuertemente las transacciones de bienes industriales entre países centrales e, incluso, tuvo lugar en gran parte de ellos un creciente proteccionismo hacia las importaciones de productos agrícolas y textiles. Este escenario atentó contra las exportaciones tradicionales de América Latina (Bértola \& Ocampo, 2013) y, aunado a las recomendaciones provenientes de la CEPAL, estimuló un cambio en la estrategia de desarrollo, que pasó a orientarse al mercado interno, sobre la base del impulso a la actividad industrial.

Pero ya, desde la década de 1970, las limitaciones del desenvolvimiento manufacturero para propiciar el cambio de las estructuras productivas de los países de la región y habilitar una forma más cualificada de inserción 
internacional (CEPAL, 1988), así como una nueva modificación en la estrategia de acumulación que comenzaba a impulsar el centro hegemónico (Kotz \& McDonough, 2010), abonaron el terreno para hacerlo propicio a que se produjera un quiebre en cuanto a las ideas y recomendaciones de políticas para el desarrollo de América Latina. La estrategia de acumulación en los centros -particularmente en el centro hegemónico, Estados Unidos- dejó de tener lugar predominantemente a través de empresas verticalmente integradas y burocráticamente administradas, que producían bienes de consumo masivo para ser realizados en espacios nacionales relativamente cerrados. La revolución tecnológica, principalmente aplicada a la informática y las telecomunicaciones, permitió que las grandes empresas industriales, llevando adelante procesos de fisión vertical, pudieran des y relocalizar su producción en múltiples y dispersos espacios geográficos, al mismo tiempo que las dotó de capacidad para articular esta diversidad de procesos que tenían lugar a escala planetaria y en tiempo real (Castells, 1990; Dicken, 2011; Gereffi \& Korzeniewicz, 1994). De este modo, las grandes empresas manufactureras, devenidas ahora en transnacionales, impulsaron un creciente outsourcing off-shore (Peck, 2017) de sus procesos productivos, descomponiendo funcional $\mathrm{y}$ espacialmente las actividades que los conforman, aunque manteniéndolas integradas en diferentes cadenas globales de producción a través de su capacidad de coordinación y comando (Fernández, 2017).

En este escenario, el pensamiento neoclásico se volvió influyente en materia de desarrollo y los Estados de diversos países periféricos dejaron de impulsar deliberadamente el proceso de industrialización. A través de procesos de privatización, desregulación y apertura, terminaron posicionando los mecanismos de mercado y la actividad privada en un lugar preponderante en la definición del desenvolvimiento económico general y de la manufactura en particular. Tuvo lugar, en palabras de Bustelo (1999), una "liberalización interna", que implicaba la desimplicación del Estado de la planificación y promoción de la actividad industrial; y una "liberalización externa", vinculada a la apertura comercial y financiera de la economía mundial. Esto significó que la estrategia de desarrollo, que desde el final de la Segunda Guerra Mundial se presentaba orientada "hacia adentro", pasara nuevamente a dirigirse hacia una participación plena en el comercio mundial. El cambio en la estrategia de desarrollo, que en los noventa pasó a ser conocida como la estrategia del Consenso de Washington (Gore, 2000), habilitó el acoplamiento de distintas actividades y espacios periféricos a las cadenas de producción global comandadas por las empresas transnacionales (ET). 
El fracaso de las políticas del Consenso de Washington (CW) en América Latina para viabilizar la salida de posicionamientos periféricos, dio lugar a una progresiva reorientación en las estrategias de desarrollo por parte de los OI. A comienzos de los años 2000, el discurso sobre el desarrollo viró hacia lo que algunos denominan un "post-CW" (Gereffi, 2014; Onis \& Senses, 2005), y emergió un nuevo lenguaje para abordar la problemática de los países periféricos. De este modo, un renovado conjunto de perspectivas teóricas $-\mathrm{y}$ estrategias de desarrollo asociadas a las mismas- fue ganando lugar en la escena internacional. Entre ellas podemos destacar los enfoques de distritos industriales, clústeres, sistemas regionales y nacionales de innovación, responsabilidad social empresaria y cooperación público-privada, por mencionar algunas. En este marco de renovación teórica emerge el enfoque de cadenas globales de valor (GVC)1, posicionado respecto de las otras perspectivas como un instrumento más holístico y potente para analizar las relaciones globales, nacionales y regionales (Kaplinsky \& Morris, 2001; Gereffi et al., 2001). Por lo indicado, tal enfoque no constituye una pieza única, sino que forma parte de un repertorio integrado por una plétora de enfoques en torno a los cuales se promueven discursos y políticas de desarrollo
(Trevignani \& Fernández, 2020). Pese a ello, el enfoque de GVC ha adquirido una fuerte influencia dentro de esta multiplicidad de perspectivas teóricas, erigiéndose en las últimas dos décadas en el dispositivo teórico dominante desde el cual pensar la nueva estrategia de desarrollo para los países periféricos.

La contribución principal del enfoque de GVC es haber advertido el cambio cualitativo en las formas de acumulación del capital en el ámbito global desde mediados de los setenta, en un intento por dar cuenta de su novedoso funcionamiento mediante investigaciones centradas en las firmas y sus relaciones con un amplio alcance sectorial, basadas en sus dos pilares: los conceptos de gobernanza y upgrading. Los estudios de la gobernanza aluden a las relaciones de poder existentes entre la firma líder y el resto de las firmas que participan en una cadena determinada. La consideración del upgrading, por su parte, hace referencia a las posibilidades que tienen las firmas no líderes que integran la cadena para escalar o mejorar sus posiciones dentro de esta2 2 .

La discusión en torno al concepto de upgrading cobró particular relevancia dentro del enfoque, debido a que paulatinamente se transformó en el foco principal de sus estudios de caso a

1 Se utiliza la sigla en inglés correspondiente a Global Value Chains debido a su difusión generalizada para referirse a este enfoque. De este modo, cuando se utilice la expresión GVC se alude al enfoque teórico; en cambio, cuando se utilicen las expresiones "cadena de producción global", "red de producción global" o similares, se hace referencia al fenómeno empírico.

2 Vale aclarar que la aproximación a estos conceptos y las problemáticas asociadas a ellos no se presenta desarticulada en la literatura de GVC, en cuanto las diferentes formas de gobernanza de las cadenas operan como uno de los condicionantes de esa movilidad ascendente. 
partir de su vinculación con la temática más general en torno al desarrollo. Esta creciente relevancia del concepto ha llevado a la clasificación de los diferentes "tipos de mejoras" que las firmas pueden alcanzar dentro de una cadena de producción determinada (Humphrey \& Schmitz, 2002). Así, se alude a los upgrading de proceso (mejorar la eficiencia de la productividad); de producto (cambiar a la confección de productos más sofisticados); funcional (adquirir nuevas capacidades o moverse a nuevos segmentos de la cadena) y, por último, el upgrading intersectorial (moverse de la cadena de una industria a otra).

Esta vinculación entre el concepto de upgrading y la problemática del desarrollo marcó un punto de inflexión en la literatura de GVC. A partir de ella, el enfoque dejó de ser considerado solamente un instrumento de análisis respecto a cómo funciona en la actualidad el sistema capitalista y comenzó a convertirse asimismo en un dispositivo teórico asociado a la problemática del desarrollo y la sugerencia de políticas públicas para alcanzarlo. La flexibilidad y la adaptabilidad de sus conceptos hicieron que gradualmente penetraran no solo en una gran cantidad de centros de estudios sobre el desarrollo de las principales instituciones académicas del mundo, sino también en una variada gama de OI. Es decir, su "borrosidad" conceptual (Stamm \& Von Drachenfels, 2011) es lo que ha permitido que ganen un lugar tanto en aquellos organismos ligados al diagnóstico y el asesoramiento -tal como los vinculados a la Organización de las Naciones Unidas-, así como también en aquellos que han operado como difusores $y$ financiadores de las estrategias de desarrollo asociadas al $\mathrm{CW}$, como el Banco Interamericano de Desarrollo (BID), el Banco Mundial (BM) o la Organización para la Cooperación y el Desarrollo Económico (OCDE).

El enfoque de GVC, en su afán por presentarse como un paradigma heterodoxo, alternativo y novedoso para la conceptualización del desarrollo del siglo XXI, lejos de retomar estrategias de desarrollo dirigido por el Estado, se ha nutrido de dos cuerpos teóricos alternativos para la sugerencia de políticas públicas. Por un lado, ha tomado en consideración planteamientos del neoinstitucionalismo, en cuanto a la necesidad de mejorar las instituciones $\mathrm{y}$ proteger la propiedad intelectual $\mathrm{y}$, por otro, del neoschumpeterianismo, en relación con el mejoramiento de la infraestructura, el apoyo a la I+D y la formación del capital humano (Wahren et al., 2018). Pese a estas modificaciones, parecen primar las continuidades sobre las diferencias con las políticas neoliberales del CW (Fernández, 2015; Neilson, 2014; Werner et al., 2014), en cuanto adopta una posición laxa frente al ingreso de IED, insiste en la reducción de barreras comerciales y plantea el fortalecimiento en la adaptación a los shocks externos. 
En el siguiente punto analizaremos la reconfiguración de las estructuras productivas periféricas en el nuevo escenario global y plantearemos las alertas que consideramos necesario encender ante la posibilidad de aceptación generalizada de esta estrategia de desarrollo para los países de la región.

\section{LA NUEVA CONFIGURACIÓN DE LA PRODUCCIÓN GLOBAL Y SUS IMPACTOS EN LAS ESTRUC- TURAS PRODUCTIVAS DE LA PERIFERIA}

Ante los límites del proceso de industrialización por sustitución de importaciones dirigido por el Estado en gran parte de América Latina, y el cambio en la estrategia estructurante de la acumulación desde el centro hegemónico, diversos países de la región -aunque con diferente nivel de intensidad- siguiendo los postulados del CW, disminuyeron los niveles de protección al comercio e incentivaron la integración a la economía mundial con base en las ventajas comparativas existentes (Bértola \& Ocampo, 2013). En este contexto tuvo lugar además una franca apertura a los flujos de capitales, lo que habilitó un significativo crecimiento de la inversión extranjera directa (IED) en la región 3 (Kerner, 2003).
La IED arribó a América Latina de la mano de las grandes empresas radicadas en los centros de la economía mundial. No obstante, es importante resaltar que la penetración del capital extranjero en la región no era una novedad, este ya había tenido una fuerte presencia en varios países durante el periodo agroexportador. Asimismo, con posterioridad tuvo una participación relevante durante el último tramo del proceso de industrialización dirigido por el Estado (Fajnzylber, 1983; Guillén Romo, 2008). Durante este período las empresas extranjeras procuraban ingresar a distintos países latinoamericanos siguiendo una estrategia de sustituir sus exportaciones a estas latitudes por producción local, sorteando así las barreras aduaneras que regulaban los flujos del comercio internacional y aprovechando la fuerte protección de los mercados internos (Fajnzylber, 1976). Sin embargo, a inicios de los años noventa, el interés que llevaba a las grandes empresas extranjeras a ingresar en América Latina ya no se encontraba exclusivamente asociado con la posibilidad de explotar sus mercados internos, estas procuraban, además, avanzar en el acoplamiento de distintas actividades que tenían lugar en diferentes países del subcontinente a cadenas de producción globales o bien regionales (Stumpo, 1998).

\footnotetext{
3 Según estadísticas de la UNCTAD, las entradas de IED en la región pasaron de aproximadamente los 8097 millones de dólares a precios corrientes en 1990, a 86020 millones de dólares a precios corrientes en 1999. Gran parte de ellas tuvo como destino el sector servicios, aunque también ha penetrado en la actividad manufacturera de distintos países de la región (Stumpo, 1998), modificando la fisonomía de la misma, como veremos en este punto del trabajo. Los flujos de IED continuaron arribando a América Latina a inicios del siglo XXI, no sin oscilaciones, promediando para el período 2000-2018 los 59588 millones de dólares a precios corrientes.
} 
En este escenario, y en consonancia con la nueva estrategia liberal de desarrollo impulsada desde Washington, América Latina profundizó el proceso de reversión industrial que venía teniendo lugar luego de abandonar la estrategia de desarrollo basada en la industrialización dirigida por el Estado. Se avanzó así en la especialización en actividades manufactureras de relativa escasa complejidad y dinamismo, beneficiadas por las ventajas comparativas estáticas -asociadas a las ventajas de los recursos naturales o a la mano de obra barata- o que han gozado de un tratamiento preferencial por parte de distintos gobiernos de la región, que las aislaba del proceso generalizado de apertura de la economía (Arceo, 2011; Bértola \& Ocampo, 2013; Katz, 2000). Solo ciertas actividades manufactureras - poco cualificadas para la generación de desarrollo tecnológico endógeno y encadenamientos productivos- resultaron parte de las estrategias de producción global o regional de las ET $^{4}$ y permitieron motorizar las economías latinoamericanas. El resto de las ramas industriales comenzaron a quedar relegadas dentro de la estructura productiva. De este modo se fueron configurando nuevas y fuertes heterogeneidades internas que profundizaron las inequidades de los distintos países de la región y agudizaron su posicionamiento periférico.
Analizando este proceso con detenimiento, se advierte que en las estructuras productivas de gran parte de los países de América Latina -fundamentalmente los del Cono Sur- adquirieron un lugar creciente las industrias productoras de alimentos y de procesamiento de recursos naturales (Arceo, 2005; 2011; Benavente et al., 1998; Katz \& Cimoli, 2001, Ocampo \& Martin, 2004) Estas actividades pasaron a formar parte de procesos de producción que operan a escala planetaria en tiempo real, con mercados de destino que transcienden el ámbito doméstico. Además, varios países de la región -como México, Colombia, Brasil y Argentina-, al amparo de un tratamiento preferencial, incrementaron también la participación de la industria automotriz dentro de la estructura productiva, aunque quedando relegada prácticamente al ensamblado de partes y piezas con tecnología importada, atendiendo al esquema de deslocalización de la producción de automóviles en el mundo (Benavente et al., 1998; Katz \& Cimoli, 2001; Katz, 2000). Por otro lado, para el caso de México y otros países de América Central y el Caribe, el bajo costo de la mano de obra, además de los beneficios impositivos y tarifarios del TLC, permitió avanzar en el ensamblado de bienes de consumo durable como computadoras, equipos de TV y video, entre otros, fundamentalmente con destino al mercado estadounidense (Arceo, 2011; Bértola

$4 \quad$ El lector interesado en estudios de caso sobre la inserción de América Latina en las cadenas de producción global pude consultar, entre otra, la siguiente bibliografía: Bair y Gereffi (2001); Giuliani et al. (2005); Mesquita y Lazzarini (2008); Quadros, (2002); Schmitz, (1999); Schteingart et al. (2017). 
\& Ocampo, 2013; Katz \& Cimoli, 2001; Ocampo \& Martin, 2004). Las zonas de las maquilas experimentaron mejoras de su desempeño en términos de crecimiento económico (Riffo, 2011), aunque aportando poco a la complejidad y el dinamismo de las estructuras productivas locales (Blyde, 2013).

Vemos así que, si bien la inserción en las cadenas globales de producción, la cual, por cierto, sin ser tan significativa en la región (Durán Lima \& Zaclicever, 2013) permitió avanzar en el proceso de industrialización (Figura 1) y en una creciente inserción exportadora sobre la base de las mismas 5 (Figura 2), estas dinámicas no bastaron para romper con la heterogeneidad y especialización estructural de los países de América Latina, ni acotar la brecha tecnológica y de ingreso existente con los países centrales. Las características de este desenvolvimiento manufacturero reforzaron la especialización en ramas de actividad industriales vinculadas a ventajas comparativas espurias (Fajnzylber, 1992), atentando contra una mayor diversificación y homogeneidad de las estructuras productivas sobre la base del desarrollo del progreso tecnológico endógeno. Ello puede advertirse al observar el escaso porcentaje de participación de manufactura de alto contenido tecnológico dentro del total de exportaciones de dicho rubro (Figura 3), el cual, a su vez, en gran parte responde a la actividad de maquila antes mencionada.

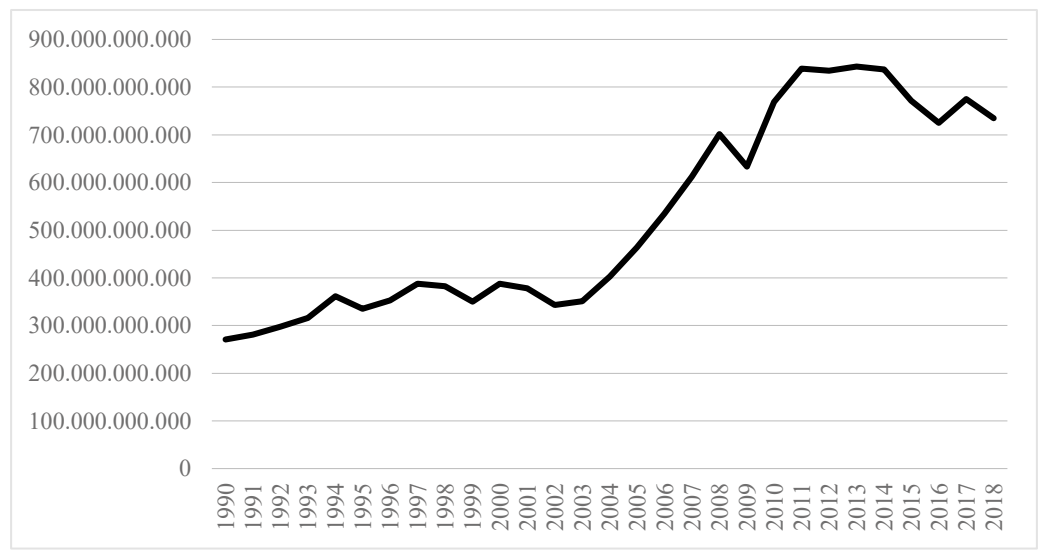

Figura 1. Evolución de la producción manufacturera de los países de América Latina a precios corrientes en dólares estadounidenses (1990-2018).

Fuente: elaboración propia sobre base de datos de United Nations National Accounts.

$5 \quad$ Nótese que este crecimiento de las exportaciones manufactureras de la región puede encontrarse sobredimensionado en virtud de la incidencia de la demanda china de bienes primarios, que habilitó el boom de los commodities (Torres González, 2018; Jenkins, 2010), e incidió significativamente en el precio de las manufacturas asociadas a los mismos (Bértola \& Ocampo, 2013). 


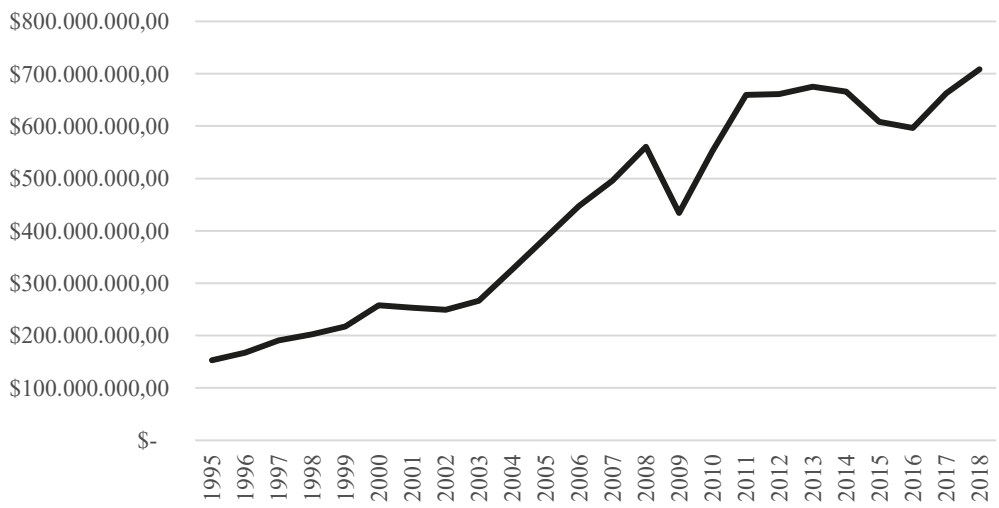

Figura 2. Evolución de las exportaciones manufactureras de América Latina en miles de dólares (1995-2018).

Fuente: elaboración propia con base en UNCTAD.

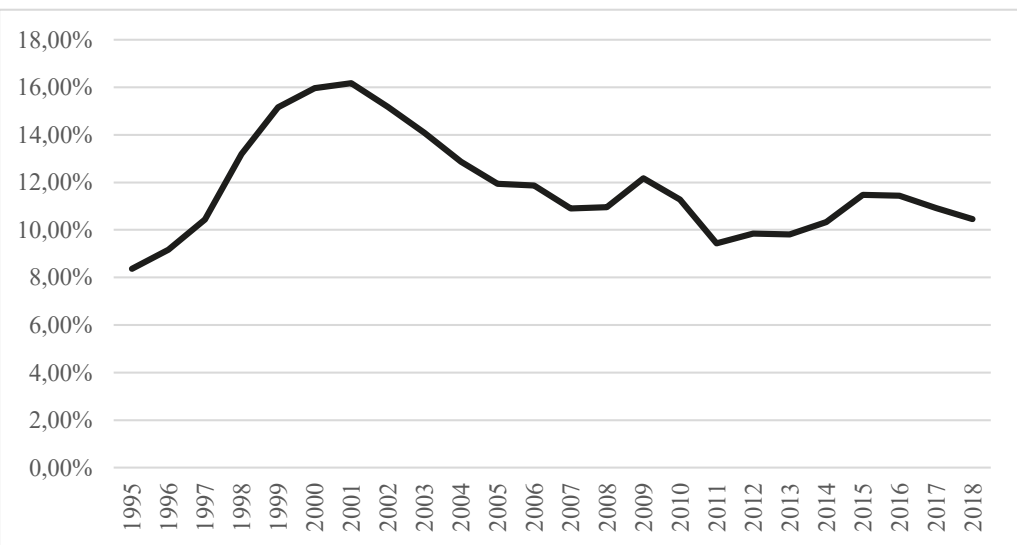

Figura 3. Evolución del porcentaje de exportaciones de manufacturas de alto contenido tecnológico de América Latina (1995-2018).

Fuente: elaboración propia con base en UNCTAD.

Ahora bien, América Latina no fue el único espacio periférico con "invitación" para industrializarse a partir de la inserción a las cadenas globales de producción. Desde finales del siglo XX y principios del siglo XXI, la producción manufacturera que históricamente permaneció concentrada en los centros del sistema capitalista mundial, con los procesos de deslocalización ha tendido a desplazarse crecientemente a países periféricos. En este escenario destaca el desempeño de un cúmulo de pequeños países localizados en Asia del Este 
que, a diferencia del resto, han podido valerse de la misma para viabilizar su salida de la periferia global.

En este contexto de reconfiguración productiva global, el este asiático ha sido la región en la que mayor dinamismo ha tenido la actividad industrial -aplanando en términos comparativos el crecimiento latinoamericano (Figura 4)- y la que más ha crecido en la producción mundial de manufacturas (Figura 5), con China convertida a inicios del siglo XXI en la mayor potencia industrial del mundo.

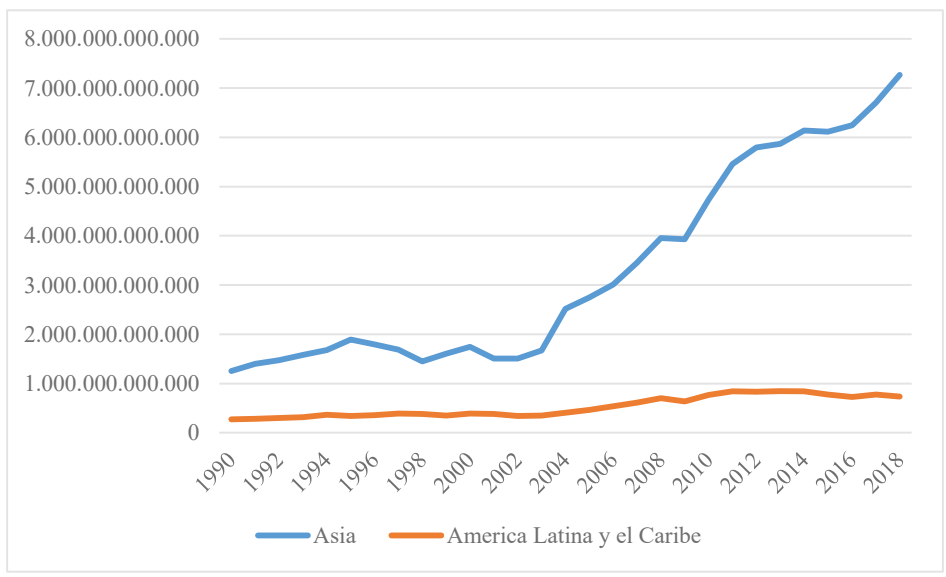

Figura 4. Evolución de la producción manufacturera de América Latina y Asia a dólares corrientes, 1990-2018.

Fuente: elaboración propia sobre base de datos de United Nations, National Accounts.

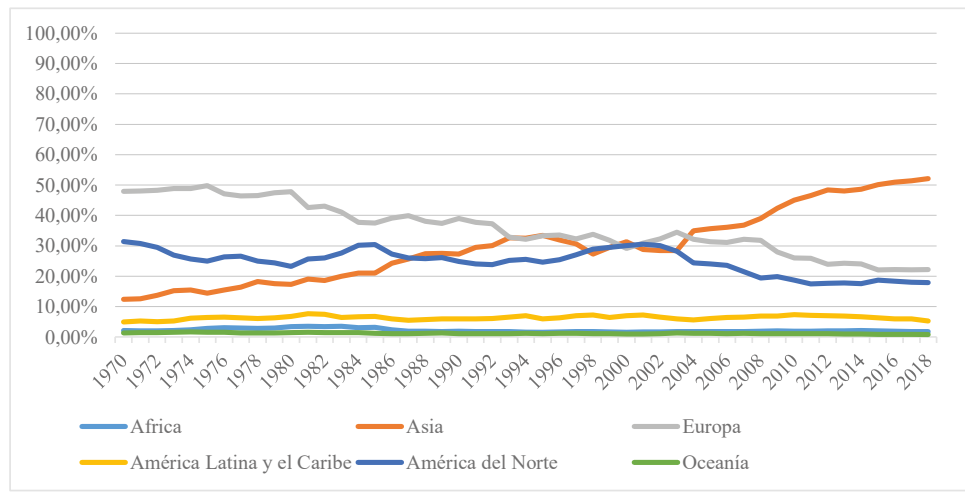

Figura 5. Evolución de la participación de las distintas regiones en la producción manufacturera mundial, 1970-2018.

Fuente: elaboración propia sobre base de datos de United Nations, National Accounts. 
Si bien el proceso de industrialización de los países asiáticos comenzó con Japón a mediados del siglo pasado, con la habilitación geopolítica de Estados Unidos en el contexto de la guerra fría (Glassman, 2018), luego fue continuado bajo la lógica del flying geese (Akamatsu, 1962), por otros países de la región (Beeson, 2009; Lemoine \& Ünal-Kesenci, 2004; Sugihara, 2019). Esta dinámica de desarrollo macrorregional se vio impulsada en gran medida por la reconfiguración de las estrategias de producción globales y la integración de estos países en cadenas globales de producción6 (Hauge, 2020; Milberg et al., 2014).

En este escenario, países como Taiwán y Corea del Sur ofrecían una mano de obra disciplinada y barata en términos internacionales y permitían llevar adelante redituablemente distintas etapas de producción manufacturera (Fröbel et al., 1977). Las primeras actividades industriales en las que se involucraron estos países no presentaban mayores niveles de sofisticación tecnológica ni requerían personal altamente calificado para su ejecución, aunque ya para 1980 fueron desarrollando actividades que demandaban mayores niveles de conocimiento, habilidades y tecnología (Gereffi, 1996).
En este proceso de upgrading dentro de las cadenas globales de producción, la política industrial implementada por los Estados desarrollistas de estos países ocupó un lugar central7 (Hauge, 2020). Esta, lejos de habilitar solamente procesos de liberalización al comercio y al capital, importó asimismo medidas proteccionistas orientadas a estimular la producción nacional, la productividad y la competitividad de las exportaciones manufactureras. En este sentido, podemos mencionar a modo de ejemplo: la orientación del crédito a la actividad industrial a través de la banca pública o mediante el uso de la banca comercial privada; el condicionamiento al ingreso de la inversión extranjera directa (IED) de las ET, que implicó vínculos de asociación con el capital local y habilitó transferencia de tecnología a las empresas nacionales, el control de componentes locales en las exportaciones, la restricción a las importaciones, tanto a través de barreras arancelarias como no arancelarias, y los subsidios a las exportaciones (Amsden, 2001; Evans, 1995; Wade, 1990).

De este modo, puede advertirse que, en contraposición a los países de América Latina, los países del este asiático que han logrado ascender en la jerarquía de riqueza dentro del sistema capitalista

$6 \quad$ La IED que recibieron los países de Asia del Este durante la última década del siglo pasado fue mayor que la que ingresó a América Latina, pasando de los 10949 millones de dólares estadounidenses a precios corrientes en 1990, a 92107 millones de dólares a precios corrientes para el año 1999, según información de la UNCTAD. EI ingreso del capital extranjero por esta vía se agudizó aún más durante los primeros años del siglo XXI, promediando entre 2000 y 2018 los 193159 millones de dólares a precios corrientes.

7 El reconocimiento de la importancia de la intervención estatal no implica desconocer la relevancia de la capacidad de articulación entre las distintas firmas asiáticas que forman parte de las cadenas de producción global y las empresas que comandan las mismas, dentro de las distintas estructuras de gobernanza, para lograr un acoplamiento estratégico en términos de desarrollo (Yeung, 2014). 
mundial, han avanzado en la configuración de un sector industrial complejo y dinámico en el escenario de reconfiguración de la producción global. En estos países, el desenvolvimiento manufacturero implicó, por un lado, avanzar en términos de innovación tecnológica, mientras que, por otro lado, posibilitó configurar un nivel significativo de complejidad de la estructura productiva asociada a la generación de eslabonamientos hacia atrás y hacia adelante, a partir de las demandas que presentaba inicialmente la "industrialización por invitación" a las cadenas globales de producción. Ello le ha permitido cualificar su participación dentro de la producción global, pasando de realizar actividades de ensamblaje de componentes impor- tados a producir domésticamente los componentes para ensamblar. Pero también, y fundamentalmente, le permitió avanzar en el desarrollo de otras actividades de la cadena de producción global, como ser la I+D, el diseño y el marketing (Gereffi, 1999). Incluso, estos países también lograron incursionar en cadenas de producción de bienes de mayor sofisticación tecnológica (Milberg et al., 2014), lo cual se evidencia en la creciente participación de bienes de medio y alto contenido tecnológico en las exportaciones de Asia. En conjunto, según resulta de la Figura 6, estas representan en promedio para el período considerado el $51.76 \%$ de los bienes exportados.

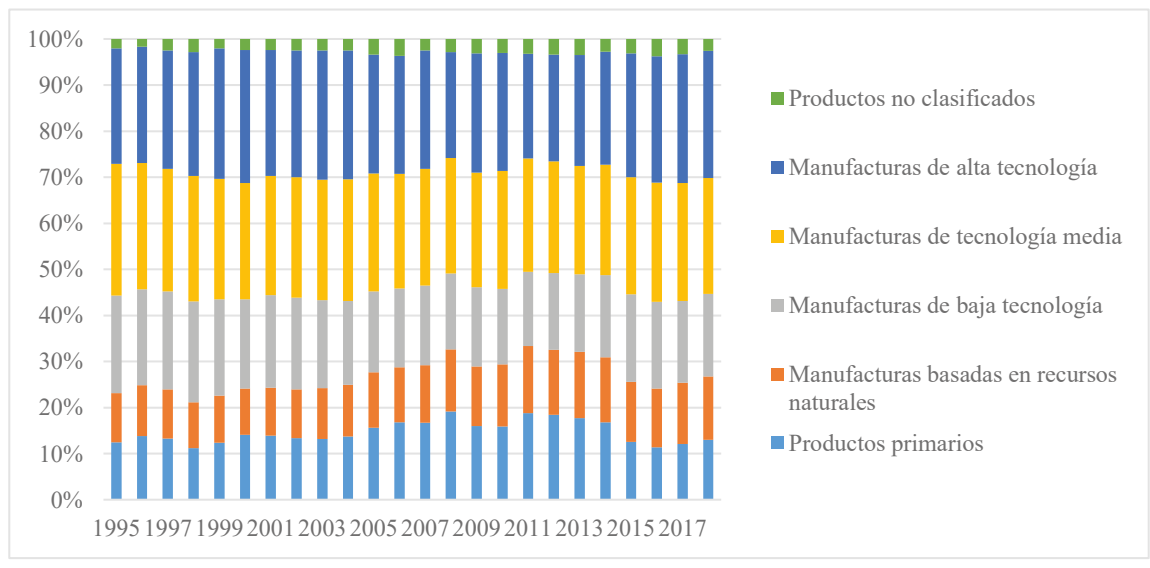

Figura 6. Evolución de la participación de los bienes de alto contenido tecnológico en las exportaciones de Asia (1995-2018).

Fuente: elaboración propia con base en UNCTAD.

En virtud de lo expuesto, las preguntas que emergen ante este escenario son: ¿hasta qué punto es recomendable adoptar una estrategia de desarrollo asociada al desenvolvimiento industrial impulsado por la inserción a cadenas globales de valor sin mayores condicionamientos? ¿Acaso no continúa siendo 
necesario, aún en el actual contexto de reproducción capitalista, diseñar e implementar una política industrial que incentive el desarrollo de una estructura productiva industrial compleja y dinámica que sirva de base para incorporarnos de una manera no subordinada a tales cadenas? En las conclusiones de este trabajo procuramos esbozar respuestas a estos interrogantes recuperando aportes del estructuralismo latinoamericano.

\section{CONCLUSIONES}

En las últimas dos décadas, el enfoque de GVC se ha consolidado no solo como un instrumento de análisis de la reconfiguración de la producción global que tuvo lugar desde la década del setenta, sino que se ha posicionado en la actualidad como el paradigma teórico para abordar la problemática del desarrollo en el mundo. Sus recomendaciones de políticas, si bien reconocen la importancia de fortalecer las instituciones y proteger la propiedad intelectual, así como mejorar las infraestructuras y el desarrollo tecnológico, lejos se encuentran de cuestionar las políticas de apertura y liberalización propias de la estrategia del CW. Esta agenda insta a un desenvolvimiento manufacturero en el marco de redes de producción global, asociado a la vieja teoría ricardiana de las ventajas comparativas, que lleva a la especialización en un segmento o actividad determinada sin que sea necesario ejecutar todas las etapas del proceso de producción del bien industrial para insertarse en el comercio mundial de manufacturas -de allí el calificativo de "industrialización fácil"-. Esta dinámica desconoce la importancia del tipo de desenvolvimiento manufacturero que tiene lugar a partir de la inserción a las redes de producción global, al mismo tiempo que no toma en cuenta el impacto que ello acarrea en la configuración de la estructura productiva de los distintos países de la periferia global. Es decir, el esquema de industrialización mediante la inserción en las cadenas productivas globales que se propone desde el enfoque de GVC, parece dejar de lado la importancia de la industrialización para la transformación de la estructura productiva de los países periféricos, tal como lo planteara el estructuralismo latinoamericano a mediados del siglo pasado.

Como se deriva de las figuras de la sección anterior, a pesar de la mayor producción y exportación manufacturera de América Latina en el marco de su integración a las cadenas globales de producción, los países de la región no han podido dejar atrás sus estructuras productivas especializadas y heterogéneas. El desenvolvimiento industrial estuvo asociado a la explotación de las ventajas comparativas -espurias-de los recursos naturales o de la mano de obra barata existentes en la región, lo que atentó contra una mayor diversificación y homogeneidad de sus estructuras productivas sobre la base del progreso tecnológico endógeno. 
En virtud de lo mencionado, se presenta entonces la necesidad de repensar el proceso de industrialización de la periferia latinoamericana para viabilizar el desarrollo en el contexto actual de cadenas globales de producción. Para ello, realizamos una recuperación actualizada de algunos de los planteamientos del estructuralismo.

En este sentido, destacamos la importancia de la manufactura, aún a inicios del siglo XXI, para la transformación estructural y la salida de posicionamientos periféricos. La actividad industrial es considerada como la correa de transmisión de los desarrollos científicos y técnicos al resto del entramado productivo. En la actividad manufacturera tienen un lugar destacado tanto los desarrollos de tecnología incorporada a los productos, como los de tecnología incorpórea. La tecnología incorporada a los productos hace referencia a la generación de bienes, particularmente de capital, producidos con tecnología de frontera. Por su parte, la tecnología incorpórea alude a cambios en las tecnologías de proceso y de producto. En función de ello, la actividad manufacturera tiene la capacidad de producir bienes y desarrollar técnicas que explican los aumentos de productividad de otros sectores -particularmente el de los servicios asociados a la produccióny permiten mantener el conjunto de la estructura productiva en la frontera tecnológica (Szirmai, 2013).
Sin embargo, como muestra la experiencia de los países que recientemente se han distanciado de la subordinación periférica, no cualquier tipo de desenvolvimiento industrial habilita procesos de transformación estructural. El proceso de industrialización que permite cualificar las estructuras productivas es aquel que tiende a configurar un complejo manufacturero complejo y dinámico (Fernández, 2017). Es decir, se trata de un proceso de industrialización que tiene la capacidad de establecer eslabonamientos intrasectoriales, que para su reproducción pone en marcha prácticas vinculadas al desarrollo de innovaciones y nuevas tecnologías.

La necesidad de avanzar en la conformación de un sector industrial complejo y dinámico para viabilizar el cambio estructural ya no responde a la lógica de llenar los distintos casilleros de la matriz insumo-producto de las economías nacionales, tal como estaba concebido el proceso de industrialización por sustitución de importaciones de los años cincuenta, cuando la acumulación de capital tenía lugar principalmente en economías nacionales relativamente cerradas. Es imperioso advertir el cambio en las lógicas de acumulación del capital que hoy operan articuladamente a través de cadenas globales de producción. Esto conlleva repensar las estrategias de política industrial.

Una política industrial que atienda esta nueva configuración productiva global demanda una combinación de instrumentos de liberalización y apertura con 
aquellos que tienden a fortalecer las instituciones y mejorar las infraestructuras y el desarrollo tecnológico de la economía en su conjunto, pero también la aplicación de instrumentos específicos de estímulo sectorial. Además de algunos elementos tradicionales de restricciones a las importaciones, como barreras arancelarias y paraarancelarias, resultan particularmente relevantes a este último respecto, tal como da cuenta la experiencia de los países asiáticos, la restricción y el control de IED de las ET, y la orientación de los excedentes canalizados en el sistema financiero hacia la actividad productiva por parte del Estado.

Asimismo, la exitosa experiencia asiática muestra la necesidad de comandar estos procesos de desenvolvimiento del sector manufacturero desde Estados nacionales que se articulen en una estrategia macrorregional, que sirva como plataforma desde la cual insertarse de manera no subordinada al nuevo escenario de producción global y que permita a través de la industrialización viabilizar el desarrollo de los países de la región.

La consideración de estas particularidades del desenvolvimiento manufacturero, imprescindibles para la transformación de las estructuras productivas de los países latinoamericanos en el nuevo escenario, así como la necesidad de apelar a distintos y forzosamente bien ensamblados instru- mentos de política industrial, nos lleva a agendar futuras investigaciones sobre el análisis de las capacidades de los Estados de la región para viabilizar la salida de la periferia global.

\section{AGRADECIMIENTOS}

Los autores forman parte del IHUCSO UNL-Conicet. Quieren agradecer a los colegas del mismo que integran el grupo de trabajo "Estado, Espacio y Desarrollo", así como también a los revisores anónimos por sus valiosos comentarios que permitieron cualificar el trabajo.

\section{CONTRIBUCIÓN DE LOS AUTORES}

Los autores participaron de manera conjunta a lo largo de la construcción del artículo.

\section{DECLARACIÓN DE CONFLICTO DE INTERESES}

Los autores declaran que no existe conflicto de intereses. Cualquier error $\mathrm{u}$ omisión es responsabilidad de los autores y no compromete a la institución.

\section{FINANCIAMIENTO}

Este trabajo no tuvo ningún tipo de financiamiento institucional. 


\section{REFERENCIAS}

[1] Akamatsu, K. (1962). A Historical Pattern of Economic Growth in Developing Countries. The Developing Economies, 1(1), 3-25. https://doi. org/10.1111/j.1746-1049.1962.tb01020.x

[2] Amsden, A. (2001). The Rise of the Rest: Challenges to the West from Late-Industrializing Economies. Oxford University Press.

[3] Arceo, E. (2005). El impacto de la globalización en la periferia y las nuevas y viejas formas de la dependencia en América Latina. Cuadernos del CENDES, 22(60), 25-61.

[4] Arceo, E. (2011). El largo camino a la crisis. Centro, periferia y transformaciones en la economía mundial. Cara o Ceca.

[5] Arrighi, G. (1999). El largo siglo XX. Akal.

[6] Arrighi, G., Silver, B., \& Brewer, B. (2003). Industrial Convergence, Globalization, and the Persistence of the North-South Divide. Studies in Comparative International Development, 38(1), 3-31.

[7] Baldwin, R. (2011). Trade and Industrialization after Globalisation'S 2nd Unbundling: How Building and Joining Supply Chains Are Different and Why it Matter. NBER Working Paper, n. w17716. National Bureau of Economic Research

[8] Bair, J., \& Gereffi, G. (2001). Local Clusters in Global Chains: The Causes and Consequences of Export Dynamism in Torreon's Blue Jeans Industry. World Development, 29(11), 1885-1903.

[9] Banco Mundial (2020). Trading for Development in the Age of Global Value Chains. World Bank.

[10] Beeson, M. (2009). Developmental States in East Asia: A Comparison of the Japanese And Chinese Experiences. Asian Perspective, 33(2), 5-39. https://www. jstor.org/stable/42704671

[11] Benavente, J. M., Crespi, G., Katz, J., \& Stumpo, G. (1998). Nuevos problemas y oportunidades en el desarrollo industrial de América Latina. En G. Stumpo (Ed.), Empresas transnacionales, procesos de reestructuración industrial y políticas económicas en América Latina. Alianza.

[12] Bértola, L., \& Ocampo, J. A. (2013). El desarrollo económico de América Latina desde la independencia. Fondo de Cultura Económica. 
[13] Blyde, J. (2013). The Participation of Mexico in Global Supply Chains. The Challenge of Adding Mexican Value. Inter-American Development Bank, Technical Note No. IDB-TN-596.

[14] Blyde, J., \& Trachtenberg, D. (2020). Global Value Chains and Latin America: A Technical Note. Inter-American Development Bank, technical note nro. 1853.

[15] Bustelo. (1999). Teorías contemporáneas del desarrollo económico. Síntesis.

[16] Castells, M. (1990). El impacto de las nuevas tecnologías en la economía internacional. Implicaciones para la economía española. Ministerio de Economía y Hacienda.

[17] CEPAL (1951). Estudio económico de América Latina, 1949. Naciones Unidas.

[18] CEPAL (1988). La industrialización en América Latina: evolución y perspectivas. Naciones Unidas.

[19] Dicken, P. (2011). Global Shift. Mapping the Changing Contours of the World Economy. The Guilford Press.

[20] Durán Lima, J. \& Zaclicever, D. (2013). América Latina y el Caribe en las cadenas internacionales de valor. CEPAL-Naciones Unidas.

[21] Evans, P. (1995). Embedded Autonomy: States and Industrial Transformation. Princeton University Press.

[22] Fajnzylber, F. (1983). La industrialización trunca de América Latina. Centro Editor de América Latina.

[23] Fajnzylber, F. (1976). Oligopolio, empresas transnacionales y estilos de desarrollo. El Trimestre Económico, 43(171), 625-656.

[24] Fajnzylber, F. (1992). Industrialización en América Latina. De la "caja negra" al "casillero vacío". Nueva Sociedad, 118, 21-28.

[25] Fernández, V. R. (2015). Global Value Chain in Global Political Networks: Tool for Development or Neoliberal Device? Review of Radical Political Economics, 47(2), 209-230. https://doi.org/10.1177/0486613414532769

[26] Fernández, V. R. (2017). La trilogía del erizo-zorro. Redes globales, trayectorias nacionales y dinámicas regionales desde la periferia. Anthropos-UNL. 
[27] Férnandez, V. R., \& Lauxmann, C. T. (2014). ¿Cuál(es) camino(s) conduce(n) a Roma? Estado y políticas industriales en los desafíos del desarrollo latinoamericano. Cuadernos del CENDES, 31(86), 49-72.

[28] FitzGerald, V. (1998). La CEPAL y la teoría de la industrialización. CEPAL, (número Extraordinario: CEPAL cincuenta años), 47-61.

[29] Fröbel, F., Heinrichs, J., \& Kreye, O. (1977). The Tendency Towards a New International Division of Labor: The Utilization of a WorldWide Labor Force for Manufacturing Oriented to the World Market. Fernand Braudel Center, 1(1), 73-88.

[30] Gereffi, G. (1996). Commodity Chains and Regional Divisions of Labour in East Asia. Jorunal of Asian Business, 12(1), 75-112.

[31] Gereffi, G. (1999). International Trade and Industrial Upgrading in the apparel Commodity Chain. Journal of International Economics, 48(1), 37-70. https://doi. org/10.1016/S0022-1996(98)00075-0

[32] Gereffi, G. (2005). The Global Economy: Organization, Governance, and Development. In N. Smelser \& R. Swedberg (Eds.), The Handbook of Economic Sociology. Second Edition (pp. 160-182). Princeton University Press.

[33] Gereffi, G. (2014). Global Value Chains in a Post-Washington Consensus World. Review of International Political Economy, 21(1), 9-37. https://doi.org/10.1080/096 92290.2012 .756414

[34] Gereffi, G., \& Korzeniewicz, M. (1994). Commodity Chain and Global Capitalism. Greenwood Publishing Group.

[35] Gereffi, G., Humphrey, J., Kaplinsky, R., \& Sturgeon, T. (2001). The Value of Value Chains: Spreading the Gains from Globalisation. IDS Bulletin, 32(3).

[36] Giuliani, E., Pietrobelli, C., \& Rabellotii, R. (2005). Upgrading in Global Value Chains :Lessons fromLatin AmericanClusters. WorldDevelopment,33(4),549-573.

[37] Glassman, J. (2018). Drums of War, Drums of Development. The Formation of A Pacific Ruling Class and Industrial Transformation in East and Southeast Asia, 1945-1980. Brill. https://doi.org/10.1163/9789004377523

[38] Gore, C. (2000). The Rise and Fall of the Washington Consensus as a Paradigm for Developing Countries. World Development, 28(5), 789-804. https://doi. org/10.1016/S0305-750X(99)00160-6 
[39] Guillén Romo, A. (2008). Modelos de desarrollo y estrategias alternativas en América Latina. En E. Correa, J. Déniz, \& A. Palezuelos (Eds.), América Latina y desarrollo económico. Estructura, inserción externa y sociedad (pp. 15-42). Akal.

[40] Gurrieri, A. (2011). O Manifesto Latino-Americano e Outros Ensaios. Contraponto.

[41] Hamilton, G., \& Gereffi, G. (2009). Global Commodity Chains, Market Makers, and the Rise of Demand-Responsive Economies. In J. Bair (Ed.), Frontiers of Commodity Chains Research. Stanford University Press.

[42] Hauge, J. (2020). Industrial Policy in the Era of Global Value Chains: Towards A Developmentalist Framework Drawing on the Industrialisation Experiences of South Korea and Taiwan. The World Economy, 00, 1-23. https://doi.org/10.1111/ twec. 12922

[43] Hirschman, A. (1968). La economía política de la industrialización a través de la sustitución de importaciones en América Latina. El Trimestre Económico, $35(140), 625-658$.

[44] Humphrey, J., \& Schmitz, H. (2002). How Does Insertion in Global Value Chains Affect Upgrading in Industrial Clusters? Regional Studies, 36(9), 1017-1027. https:// doi.org/10.1080/0034340022000022198

[45] Jenkins, R. (2010). China's Global Expansion and Latin America. Journal of Latin American Studies, 42(4), 809-837

[46] Julio, L.G. (2008). Keynes in Latin America and Latin American Keynesianism. En: Forstater M., Wray L.R. (eds) Keynes for the Twenty-First Century. Palgrave Macmillan.

[47] Kaplinsky, R., \& Morris, M. (2001). A Handbookfor Value Chain Research. IDRC.

[48] Katz, J. (2000). Reformas estructurales, productividad y conducta tecnológica en América Latina. Fondo de Cultura Económica- CEPAL.

[49] Katz, J., \& Cimoli, M. (2001). Reformas estructurales, brechas tecnológicas y el pensamiento del Dr. Raúl Prebisch. CEPAL, Serie Desarrollo Productivo y Empresarial.

[50] Kerner, D. (2003). La CEPAL, las empresas transnacionales y la búsqueda de una estrategia de desarrollo latinoamerica. CEPAL, 79, 85-99. 
[51] Kotz, D., \& McDonough, T. (2010). Global Neoliberalism and the Contemporary Social Structure of Accumulation. In T. McDonough, M. Reich, \& D. Kotz (Eds.), Contemporary Capitalism and its Crises: Social Structure of Accumulation Theory for the 21st Century. Cambridge University Press.

[52] Lemoine, F. \& Ünal-Kesenci, D. (2004). Assembly Trade and Technology Transfer: The Case of China. World Development, 32(5), 829-850. https://doi. org/10.1016/j.worlddev.2004.01.001

[53] Mesquita, L., \& Lazzarini, S. (2008). Horizontal and Vertical Relationships in Developing Economies: Implications for SMEs' Access to Global Markets. Academy of Management Journal, 51(2), 359-380.

[54] Milberg, W., Jiang, X. \& Gereffi, G. (2014). Industrial Policy in the Era of Vertically Specialized Industrialization. In J. M. Salazar-Xirinachs, I. Nübler, \& R. Kozul-Wright (Eds.), Transforming Economies: Making Industrial Policy Work for Growth, Jobs and Development. International Labour Organization.

[55] Nahón, C., Rodríguez Enríquez, C., \& Schorr, M. (2006). El pensamiento latinoamericano en el campo del desarrollo del subdesarrollo: trayectoria, rupturas y continuidades. CLACSO.

[56] Neilson, J. (2014). Value Chains, Neoliberalism and Development Practice: The Indonesian Experience. Review of International Political Economy, 21(1), 38-69. https://doi.org/10.1080/09692290.2013.809782

[57] Ocampo, J. A. \& Martin, J. (2004). América Latina y el Caribe en la era global. Alfaomega.

[58] Onis, Z., \& Senses, F. (2005). Rethinking the Emerging Post-Washington Consensus. Development and Change, 36(2), 263-290. https://doi. org/10.1111/j.0012-155X.2005.00411.x

[59] Peck, J. (2017). Offshore: Exploring the Worlds of Global Outsourcing. Oxford University Press.

[60] Prebisch, R. (1949). El desarrollo económico de América Latina y sus principales problemas. CEPAL.

[61] Quadros, R. (2002). Global Quality Standards, Chain Governance and the Technological Upgrading of Brazilian Auto-Components Producers. IDS Working Paper 156. 
[62] Riffo, L. (2011). Desigualdades económicas regionales en América Latina y el Caribe. En CEPAL Reuniones de Expertos sobre Población, Territorio y Desarrollo Sostenible.

[63] Rodríguez, O. (2006). El estructuralismo latinoamericano. Siglo XXI-CEPAL.

[64] Schmitz, H. (1999). Global Competition and Local Cooperation: Success and Failure in the Sinos Valley, Brazil. World Development, 27(9), 1627-1650.

[65] Schteingart, D., Santarcángelo, J., \& Porta, F. (2017). La inserción Argentina en las cadenas globales de valor. Asian Journal of Latin American Studies, 30(3), 45-82.

[66] Sugihara, K. (2019). The Asian Path of Economic Development: Intra-regional Trade, Industrialization and the Developmental State. In T. Shiraishi and T. Sonobe (Eds.), Emerging States and Economies. The Origins, Drivers, and Challenges Ahead. Springer.

[67] Stamm, A., \& Von Drachenfels, C. (2011). Value Chain Development: Approaches and Activities by Seven UN Agencies and Opportunities for Interagency Cooperation. International Labour Office.

[68] Stumpo, G. (1998). Empresas transnacionales, procesos de reestructuración industrial y políticas económicas en América Latina. Alianza.

[69] Svampa, M. (2016). Debates latinoamericanos. Indianismo, desarrollo, dependencia y populismo. Edhasa.

[70] Szirmai, A. (2013). Manufacturing and Economic Development. In A. Szirmai, W. Naudé, \& L. Alcorta (Eds.), Pathways to Industrialization in the Twenty-First Century. New Challenges and Emerging Paradigms. Oxford University Press.

[71] Torres González, J. (2018). El Pacífico suramericano y su integración con China y Asia. Apuntes del Cenes, 37(65), 77-116. https://doi.org/10.19053/01203053.v37. n65.2018.5886

[72] Trevignani, M., \& Fernández, V. (2020). The Institutional Process of the GVC Approach: From The Academic Field to the International Organizations. In S. Faust (Ed.), Understanding Value Chains. Nova Science Publishers.

[73] Unceta Satrústegui, K. (2009). Desarrollo, subdesarrollo, maldesarrollo y postdesarrollo. Carta Latinoamericana, 7, 1-34. 
[74] Wade, R. (1990). Governing the Market: Economic Theory and the Role of the Goverment in East Asian Industrialization. Princeton University Press.

[75] Wahren, P., Cúneo, D., Di Giovambattista, A., \& Gárriz, A. (2018). Cadenas globales de valor: la reinterpretación de los organismos internacionales. Realidad Económica, 314 (47), 37-69.

[76] Wallerstein, I. (1993). The Geoculture of Development, or Transformation of our Geoculture? Asian Perspective, 17(2), 211-225.

[77] Werner, M., Bair, J. \& Fernández, V. (2014). Linking Up to Development? Global Value Chains and the Making of a Post Washington Consensus. Development and Change, 45(6), 1219-1247. https://doi.org/10.1111/dech.12132

[78] Yeung, H. W. C. (2014). Governing the Market in A Globalizing Era: Developmental States, Global Production Networks and Inter-Firm Dynamics in East Asia. Review of International Political Economy, 21(1), 70-101. https://doi.org/10.1080/0969229 0.2012 .756415 